\title{
Role of Tissue Markers on Predicting the Prognosis of Intermediate and High-risk Non-muscle Invasive Bladder Cancer
}

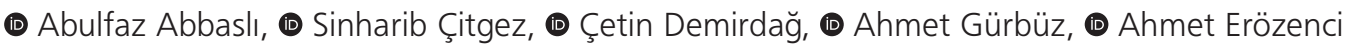 \\ Istanbul University-Cerrahpaşa, Cerrahpaşa Faculty of Medicine, Department of Urology, Istanbul, Turkey
}

\begin{abstract}
Objective: Investigating the recurrence of high-risk non-muscle invasive bladder cancer patients with the phosphohistone H3 (PPH3), P53, and Ki-67 as a predictive markers.

Materials and Methods: A total of 46 patients with high-risk non-muscle invasive bladder cancer patients were included. All the patients treated with bacillus Calmette-Guérin instillation. Patients were divided into two groups consisted of the with recurrence (group 1) and without recurrence (group 2). Assessment of mitotic cells only in metaphase that are stained with PPH3 immunhistochemically counted separately at 20 and 40 magnification in most intense areas under microscope. Immunhistochemically stained urothelial cells with P53 evaluation was based on 3 different scoring categories that are intense, extent, and the total score. Ki-67 immunhistochemical stained cells were calculated with ratio of counted positive stained cells in intense area to the total cells. Mann-Whitney $U$ test was used.

Results: There is no significant differences between the median value of PPH3 at 20 and 40 magnification in the two groups ( $p=0.620, p=0.241)$. P53 that was assessed with median values of the extent, intense, and total score had no significant findings between the two groups $(p=0.377, p=0.102, p=0.380)$. Also, no significant results in median values of $\mathrm{Ki}-67$ between the two groups found.

Conclusion: These markers could not predict recurrence in our study, but in our opinion, significant results will be found if increasing case numbers and standard immunhistochemichal staining and assessment methods are used.
\end{abstract}

Keywords: Bladder cancer, marker, recurrence

\section{Introduction}

Approximately $80 \%$ of transitional epithelial cell cancers of the bladder are limited to the mucosa and submucosa at the time of diagnosis. This condition is called superficial bladder tumor or non-muscle invasive bladder cancer (NMIBC). NMIBC is a very heterogeneous group, with papillary urothelial tumors confined to the mucosa, defined as low grade Ta on one side of the spectrum, and high grade T1 tumors with carcinoma in situ (CIS) on the other. Approximately $70 \%$ of NMIBCs are $\mathrm{Ta}, 20 \% \mathrm{~T} 1$, and $10 \% \mathrm{CIS}$ lesions. The prognosis of NMIBC is generally good, but recurrence is seen in $30-80 \%$ of patients, while muscle invasive disease develops within 5 years in $1-45 \%$ of patients $(1,2,3)$.
NMIBC is a lifelong chronic disease that often tends to relapse, and sometimes progresses. The aim of its treatment is to eliminate the disease, to prevent relapse and progression. There have been many studies on prognostic factors to predict the likelihood of relapse and many studies continue to be done. Currently, the two most important prognostic factors in this regard are tumor stage and grade, but these two prognostic factors alone are insufficient to determine the prognosis of NMIBC. Therefore, the investigation of tissue markers that can be used to predict the recurrence of bladder cancer comes to the fore.

Histone $\mathrm{H} 3$ is one of the histone proteins that make up the chromatin, and it has recently been reported that it is closely related to mitosis and that the anti-phosphohistone $\mathrm{H} 3$ ( $\mathrm{PPH} 3$ )

Cite this article as: Abbaslı A, Çitgez S, Demirdağ Ç, Gürbüz A., Erözenci A. Role of Tissue Markers on Predicting the Prognosis of Intermediate and High-risk Nonmuscle Invasive Bladder Cancer. Bull Urooncol 2020;19(4):177-181

Address for Correspondence: Sinharib Çitgez, İstanbul University-Cerrahpaşa, Cerrahpaşa Faculty of Medicine, Department of Urology, İstanbul, Turkey Phone: +90 5323122377 E-mail: drsinharib@yahoo.com ORCID-ID: orcid.org/0000-0002-3897-2951 Received: 15.09.2019 Accepted: 02.01.2020 
antibody is a mitosis specific antibody. In the literature, it has been shown that PPH3 is used in grading of other cancer types such as brain and breast $(4,5,6,7,8)$. Ki-67 protein expression is highly associated with cell proliferation and is present in all phases of the cell cycle (G1, S, G2 and mitosis) except G0. It is an excellent marker that determines the growth fraction of the cell population of the $\mathrm{Ki}-67$ protein. In the literature, fractions of Ki-67 positive tumor cells have been associated with clinical progression, and it has been shown to have prognostic value in terms of survival and tumor recurrence in prostate and breast cancer (9).

P53 tumor suppressor gene has been shown in previous studies to play an important role in carcinogenesis. The most common genetic abnormality in other malignancies, including bladder cancer, is the P53 mutation. It has been found in the literature that changes in P53 are associated with bladder cancer progression, and it has been shown in recent studies that it can be used as a predictive factor for disease-related survival and disease-independent survival (10).

In this study, we aimed to investigate whether PPH3, Ki-67 and P53 are predictive markers in determining recurrence in patients with high-risk NMIBC.

\section{Materials and Methods}

In the study, the data of patients who underwent transurethral tumor resection for NMIBC between 2008 and 2015 in İstanbul University-Cerrahpaşa, Faculty of Medicine, Department of Urology were retrospectively reviewed. Risk stratification was made according to the European Association of Urology guidelines (2). Accordingly, 46 patients diagnosed as having high-risk NMIBC were included in the study. The first resection preparations of all patients were evaluated. The cystoscopic follow-up protocol of the patients was applied every three months for the first 2 years, every six months for the next 3 years, and then annually, if there was no recurrence after the first resection. Recurrence at least once in the follow-up of the patients was accepted as recurrence. Bacillus Calmette-Guérin treatment was applied to all patients. Patients who received 6 months of induction and at least 1 year of maintenance therapy were included in the study. The patients included in the study were divided into two groups according to their recurrence status as the group with recurrence (group 1) and the group without recurrence (group 2).

PPH3, Ki-67 and P53 results were re-evaluated by a single pathologist by performing manual immunohistochemical staining in the first resection pathology specimens of both groups. The slides of the specimens stained immunohistochemically with PPH3 were evaluated under the microscope by counting only mitosis cells in the metaphase phase at 20 and 40 magnifications under the microscope. Urothelial cells immunohistochemically stained with P53 were evaluated in three different scoring categories as intensity, prevalence and total score. Intensity evaluation according to the staining intensity of the cells were: No staining (score 0 ), weak staining (score 1 ), moderate staining (score 2 ), and severe staining (score 3 ). The prevalence scoring according to the proportion of positive cells stained were: $0 \%$ (score 0 ), $1-10 \%$ (score 1), 10-25\% (score 2), 25-
$50 \%$ (score 3 ), $50-75 \%$ (score 4 ), $>75 \%$ (score 5 ). The total score of the patients was calculated by multiplying the extent and intensity scores (lowest score: 0 and highest score: 15). Ki67 immunohistochemical staining, also known as proliferation marker, was determined by calculating the ratio of positive cells stained in the most intensive area to total cells.

The results of both groups were compared by applying the Mann-Whitney $U$ test. A p-value of less than 0.05 was considered significant. The study was approved by the Ethics Committee of Cerrahpaşa Medical Faculty.

\section{Statistical Analysis}

Statistical analyzes were performed using the Statistical Package for the Social Sciences (SPSS) version 21.0 software (IBM SPSS Statistics for Windows, version 21.0. Monk, NY: IBM Corp., Armonk, NY). Pearson chi-square and Fisher's Exact tests were used to search for the difference between categorical variables, and Kruskal-Wallis and Mann-Whitney $U$ tests were used to search for the difference between means. A p-value of less than 0.05 was considered statistically significant. The study protocol was approved by the Cerrahpaşa Medical Faculty Ethics Committee (83045809/604.01/02-218946). The consent form was filled in by all participants and their families.

\section{Results}

A total of 46 patients were included in the study and 23 of these patients were included in group 1, while the other 23 were included in group 2.

Three (13\%) of the patients belonging to group 1 were female and $23(87 \%)$ were male. Mean age was determined as $72.1 \pm 11.6$ (53-85). Average follow-up period was calculated as $43.2 \pm 8.6$ months. Ten of the patients (43.4\%) had Ta high grade, 13 (56.6\%) had T1 high grade pathology. CIS Ta was found in the pathological diagnosis of 2 patients with high grade.

Three (13\%) of the patients belonging to group 2 were female and $23(87 \%)$ were male. Mean age was determined as $66.8 \pm 4.9$ (40-81). Mean follow-up period was calculated as $56.5 \pm 11.4$ months. The number of patients with a diagnosis of Ta high grade was $14(60 \%)$, and the number of patients with a diagnosis of T1 high grade was 9 (40\%). Three patients (13\%) with Ta high grade were diagnosed as having CIS (Table 1).

In group 1, the mean values of PPH3 of mitosis cells counted at 20 and 40 magnification were determined as $11.5(1-27)$ and 6.1 (1-12). The mean P53 intensity value was 2 (1-3), the mean prevalence value was 1.7 (1-5), and the total score mean value was 4.1 (1-15). Finally, the average result of Ki-67 was calculated as $13 \%(3-30 \%)$.

In group 2, the mean values of PPH3 of mitosis cells counted at 20 and 40 magnification were found as $14.3(1-48)$ and 7.6 (120). The mean P53 intensity value was $1.6(0-3)$, the prevalence mean value was $2.6(0-59)$, and the total score mean value was 6.1 (0-15). The mean value of $\mathrm{Ki}-67$ was found to be $13 \%$ (2$30 \%$ ) (Table 2).

When the mean values of both groups were compared, no significant difference was found in PPH3 at magnification of 20 


\begin{tabular}{|c|c|c|c|}
\hline & Group 1 & Group 2 & $\mathrm{p}$ \\
\hline Mean age & $72.1 \pm 11.6$ & $66.8 \pm 4.9$ & 0.11 \\
\hline \multicolumn{4}{|l|}{ Gender } \\
\hline Female & $3(13 \%)$ & $3(13 \%)$ & $>0.999$ \\
\hline Male & $20(87 \%)$ & $20(87 \%)$ & - \\
\hline Mean follow-up (months) & $43.2 \pm 8.6$ & $56.5 \pm 11.4$ & 0.55 \\
\hline \multicolumn{4}{|l|}{ T stage } \\
\hline $\mathrm{Ta}$ & $10(43.4 \%)$ & $14(60 \%)$ & $>0.99$ \\
\hline T1 & $13(56.6 \%)$ & $9(40 \%)$ & $>0.99$ \\
\hline $\mathrm{Ta}+\mathrm{CIS}$ & $2(8.6 \%)$ & $3(13 \%)$ & $>0.99$ \\
\hline
\end{tabular}

Table 2. As a result of statistical analysis, it was determined that PPH3, Ki-67, P53 could not be used as predictive molecular markers to determine recurrence

\begin{tabular}{|c|c|c|c|}
\hline & Group 1 & Group 2 & $p$ \\
\hline \multicolumn{4}{|l|}{ PPH3 } \\
\hline ×20 magnification & $11.5(1-27)$ & $14.3(1-48)$ & 0.620 \\
\hline x40 magnification & $6.1(1-12)$ & $7.6(1-20)$ & 0.241 \\
\hline \multicolumn{4}{|l|}{ P53 } \\
\hline Intensity & $2(1-3)$ & $1.6(0-3)$ & 0.377 \\
\hline Prevalence & $1.7(1-5)$ & $2.6(0-5)$ & 0.102 \\
\hline Total score & $4.1(1-15)$ & $6.1(0-15)$ & 0.380 \\
\hline Ki-67 & $13 \%(3-30)$ & $13 \%(2-30)$ & 0.757 \\
\hline
\end{tabular}

and $40(p=0.620, p=0.241)$. P53 prevalence, intensity and total score mean value were not significantly different between both groups ( $p=0.377, p=0.102, p=0.380$ ). Ki-67 mean values were not significantly different between the two groups $(p=0.757)$.

\section{Discussion}

Bladder cancers are a heterogeneous group of diseases and there are many factors affecting recurrence, progression and survival. Routinely used tumor characteristics such as tumor stage and grade have limited effects on routine clinical practice, therefore, it has prompted the investigation of molecular markers to predict tumor recurrence and progression (11). In this study, we investigated whether PPH3, P53 and Ki-67, which were molecular markers each in patients with NMIBC, could be used as a predictive marker to predict recurrence.

In the statistical analysis, when the average values of group 1 and group 2 were compared, no significant difference was found in PPH3 at 20 and 40 magnification $(p=0.620, p=0.241)$. P53 prevalence, intensity and total score mean value were not significantly different between both groups $(p=0.377, p=0.102$, $\mathrm{p}=0.380$ ). Ki-67 mean values were not significantly different between the two groups $(p=0.757)$. As a result of the study, it was concluded that these markers could not predict recurrence.

PPH3 is the core histone protein of eukaryotic cell chromatin. While PPH3 is not seen at all in the intraphase phase, it reaches its maximum levels in the chromatin condensation phase of mitosis. Anti-PPH3 is specific for phosphorylated $\mathrm{H} 3$ and is used as a mitosis marker. Its use as a marker of mitosis in many different tumor types such as breast cancer, malignant melanoma, meningioma, and lung cancer has been validated $(6,7,8,9,10)$. Related to this, there is $50-60 \%$ disagreement between pathologists in the grading of upper urinary tract and bladder urothelial cancers. Therefore, Solomides et al. (12) investigated whether PPH3 can be used as a molecular marker that could eliminate this variability in the grading of upper urinary system urethral cancers in their study conducted in 2012. Sixty one patients with upper urinary tract urothelial cancer were included in that study. Pathology specimens of the patients were stained with both Hematoxylin \& Eosin (H\&E) and PPH3 immunohistochemical staining with $\mathrm{H} \& \mathrm{E}$. Tumor grades were compared with the 1973 World Health Organization (WHO) classification (1: well differentiated, 2: moderately differentiated, 3: poorly differentiated) used in the grading of tumors with this method. The results were evaluated by three pathologists. Eighty percent consensus was achieved in the grading of tumors after staining the cell blocks with $\mathrm{PPH} 3$ together with $\mathrm{H \& E}$, while this ratio was found to be $55 \%$ with the use of the 1973 WHO classification. In another study, Gunia et al. (13) compared Ki67 and PPH3 values in the differentiation of $\mathrm{CIS}$ and carcinoma non-superficial tumors. The study included $32 \mathrm{CIS}, 31$ non-CIS superficial lesions (low grade dysplasia and reactive atypia). Differentiation of superficial lesions with and without CIS was made according to the 2004 WHO classification. As a result of that study, it was found that the rate of cells stained positive with $\mathrm{Ki}-67$ was higher in patients with CIS. It was shown that it could be used as an important marker in the differentiation of patients with and without $\mathrm{CIS}$. When looking at PPH3 values, another molecular marker used to differentiate superficial bladder tumors with and without $\mathrm{CIS}$, no significant difference was found between the two groups ( $p>0.25)$. Parallel to this, when the mean values of both groups were compared in our study, no significant difference was found in terms of PPH3 at magnification of 20 and $40(p=0.620, p=0.241)$.

Mangrud et al. (14) investigated whether Mitotic Activity index (MAl), Ki-67 and PPH3 markers had superiority over the 19732004 WHO classification in predicting prognosis in NMIBC in a study conducted in 2013. Two hundred forty nine patients were included in that study, 125 of those patients had highrisk NMIBC and 124 had moderate-risk or low-risk NMIBC. In that study, the most intense proliferation area was selected in the evaluation of PPH3 staining and the evaluation was made by counting the mitosis cells in this area. MAI was selected by selecting the area where proliferation was the most intense at low magnification under the microscope of the specimens painted with H\&E and the mitotic structures were counted at 400 magnification in a total area of $1.59 \mathrm{~mm}^{2}$. Whether those three proliferation markers could be used to predict progression were investigated. The patients were divided into two groups as patients with and without progression, and their average results were compared. The mean value of PPH3 was found as 42 in the group with progression, 17 in the non-progressive group $(p=0.31)$, and the mean value of Ki-67 as $40 \%$ in the group with progression and $16 \%$ in the non-progressive group $(p=0.006)$. The mean value of the MAI was shown to be statistically 
significantly superior in predicting progression compared to the mean values of the other two proliferation markers $(p<0.001)$. As a result of the study, it was stated that PPH3 and $\mathrm{Ki}-67$ alone could be used as prognostic markers related to progression, but it was concluded that they did not add additional prognostic value to MAI in the multivariate model.

Apart from the mentioned studies, studies on whether PPH3 can be used as a molecular marker in bladder cancer are very limited. In our study, when the mean values of the number of mitosis cells stained with PPH3 were compared, no significant difference was found between the two groups. In our study, unlike the study conducted by Mangrud et al. (14), only patients diagnosed as having high-risk NMIBC were included and the results of the patients were evaluated by a single uropathologist. While Mangrud et al. (14) showed that PPH3 alone did not contribute to progression as a prognostic marker, in addition to this, it was determined that PPH3 could not be used as a marker to predict recurrence in our study.

It is known that the most frequently mutated gene in patients with cancer is the P53 tumor suppressor gene. P53 gene mutation in different tumor types has been demonstrated by immunohistochemical staining. Studies have shown that P53 gene mutation correlates with progression, but studies indicating the opposite are also in the literature $(15,16)$. In the literature, Abdelzaher and Kotb (17) investigated whether runt-related transcription factor-2 (RUNX2) and P53 were independent markers to predict recurrence of bladder cancers in 2014. A total of 87 patients with a diagnosis of MIBC together with primary NMIBC were included in that study. As a control group, sampling was made from the bladder mucosa of 17 patients without neoplasia. The patients were followed prospectively for an average of $\mathbf{2 8 . 8}$ months and their results were evaluated. The probability of predicting recurrence was found to be $40.9 \%$ for P53. When P53 value was considered together with RUNX2 in predicting recurrence, it was shown that both of them could accurately predict excessive expression and recurrence rate of $73.6 \%$. However, it would not be correct to conclude that P53 could only be used as a predictive marker to show recurrence in patients diagnosed as having high-risk NMIBC due to the inclusion of patients with a diagnosis of MIBC with a high probability of recurrence and progression in that study. In our study, the mean P53 intensity value in group 1 was calculated as 2 (1-3), the mean prevalence value as 1.7 (1-5), and the total score mean value as 4.1 (1-15). In group 2, the mean P53 intensity value was $1.6(0-3)$, the average prevalence value was 2.6 (0-59), and the total score mean value was 6.1 (0$15)$. In the statistical analysis, P53 prevalence, intensity and total score mean values were not found to be different between both groups ( $p=0.377, p=0.102, p=0.380)$.

Malats et al. (16) reviewed a total of 117 studies about whether P53 was a prognostic marker for bladder cancer in the meta-analysis in 2005. In only 9 (27\%) of 34 studies on recurrence, P53 overexpression was found to have a prognostic value in showing recurrence. The reasons for this were that the pathological characteristics of the patients included in the studies were heterogeneous, the number of patients was below 100 in 82 (70\%) studies and the absence of standard immunohistochemistry staining, scoring strategy, and positive staining definition. The result about P53 in our study shows parallelism with the result of this meta-analysis.

$\mathrm{Ki}-67$ is proliferation-associated nuclear antigen and is expressed in the entire cell cycle except $\mathrm{G} 0$. Ki-67 protein is widely used as a proliferation marker reflecting the number of tumoral cell cycles (18). In some studies in the literature, the correlation of Ki-67 with progression was not observed, and it was shown that it was closely related with recurrence $(19,20)$.

Ben Abdelkrim et al. (21) investigated the utility of topoisomerase 2 alpha, Ki-67 and P53 expression in predicting recurrence and progression in low-, moderate- and high-risk NMIBC in 2014. Seventy one patients were included in the study, and 69 patients were evaluated. Average follow-up period was calculated as 28 months. During the follow-up, 34 patients did not have recurrence, 20 patients had recurrence and 15 patients had progression. In that study, Ki-67 positive value was accepted as $>10 \%$. The number of Ki-67 positive patients was 27 (38\%), and it was concluded that it was associated with recurrence. However, the relationship of $\mathrm{Ki}-67$ with progression could not be demonstrated. In the study, only 19 of 69 patients were diagnosed as having high-grade NMIBC, and only $8(42.1 \%)$ of these 19 patients were found to have a positive Ki-67 value (>10\%). In our study, the Ki-67 positive value of patients with high-grade NMIBC was not determined in the evaluation of recurrence, and the mean values of both groups (group 1: $13 \%$ and group 2: $13 \%$ ) were compared. The $>10 \%$ method, which Ben Abdelkrim et al. (21) determined as the positive value of Ki-67, was shown to be positive in 14 (60\%) patients in the group without relapses and in 9 (40\%) patients in the group with relapses when applied in our study. Ki-67's status related to recurrence in the study of Ben Abdelkrim et al. (21) is in contradiction with the result of our study. The reason for this can be explained by the short mean patient follow-up period (28 months) and the presence of patients with NMIBC with heterogeneous pathological features (neoplasm with low malignancy potential, low-grade malignancy and high-grade malignancy).

\section{Study Limitations}

Our study had some limitations. The first was that it was retrospective. Second, the number of patients was limited. For all these reasons, prospective studies that would be conducted in the future might eliminate these deficiencies. Nevertheless, considering the limited number of studies in the literature on this subject, it can be thought that our study will contribute to the literature.

\section{Conclusion}

In this study, it was determined that PPH3, Ki-67, P53 could not be used as predictive molecular markers in determining recurrence in patients with high-risk NMIBC. Standard evaluation methods can only be developed in the presence of standard immunohistochemical staining methods. Other molecular markers are needed that can be used to predict the recurrence and progression of bladder tumors. There is a need for prospective and high quality studies on this subject. 


\section{Acknowledgements}

Publication: The results of the study were not published in full or in part in form of abstracts.

Contribution: There is not any contributors who may not be listed as authors.

Conflict of Interest: No conflict of interest was declared by the authors.

Financial Disclosure: The authors declared that this study received no financial support.

\section{Ethics}

Ethics Committee Approval: The study protocol was approved by the Cerrahpaşa Medical Faculty Ethics Committee (83045809/604.01/02-218946).

Informed Consent: The consent form was filled in by all participants and their families.

Peer-review: Externally peer-reviewed.

\section{Authorship Contributions}

Concept: A.A., S.Ç., Design: S.Ç., Ç.D., A.E., Data Collection or Processing: A.G., Ç.D., Analysis or Interpretation: S.Ç., A.E., Ç.D., Literature Search: A.A., Ç.D., A.E., Writing: S.Ç., Ç.D., A.A.

\section{References}

1. Soloway MS, Sofer M, Vaidya A. Contemporary management of stage $\mathrm{T} 1$ transitional cell carcinoma of the bladder. J Urol 2002; 167:1573-1583.

2. Babjuk M, Oosterlinck $W$, Sylvester $R$, et al. EAU guidelines on non-muscle-invasive urothelial carcinoma of the bladder, the 2011 update. Eur Urol 2011;59:997-1008.

3. van Rhijn BW, Burger M, Lotan $Y$, et al. Recurrence and progression of disease in non-muscle-invasive bladder cancer: from epidemiology to treatment strategy. Eur Urol 2009;56:430-442.

4. Hendzel MJ, Nishioka WK, Raymond Y, et al. Chromatin condensation is not associated with apoptosis. J Biol Chem 1998;273:24470-24478.

5. Hendzel MJ, Wei Y, Mancini MA, et al. Mitosis-specific phosphorylation of histone $\mathrm{H} 3$ initiates primarily within pericentromeric heterochromatin during $\mathrm{G} 2$ and spreads in an ordered fashion coincident with mitotic chromosome condensation. Chromosoma 1997; 106:348-360.

6. Zbytek B, Cohen C, Wang J, et al. Nottingham-defined mitotic score: comparison with visual and image cytometric phosphohistone $\mathrm{H} 3$ labeling indices and correlation with Oncotype DX recurrence score. Appl Immunohistochem Mol Morphol 2013;21:48-53.
7. Nielsen PS, Riber-Hansen R, Jensen TO, et al. Proliferation indices of phosphohistone $\mathrm{H} 3$ and Ki67: strong prognostic markers in a consecutive cohort with stage $1 /$ II melanoma. Mod Pathol 2013;26:404-413.

8. Ribalta $\mathrm{T}$, McCutcheon IE, Aldape $\mathrm{KD}$, et al. The mitosis-specific antibody anti-phosphohistone- $\mathrm{H} 3$ ( $\mathrm{PHH} 3)$ facilitates rapid reliable grading of meningiomas according to WHO 2000 criteria. Am J Surg Pathol 2004;28:1532-1536.

9. Scholzen T, Gerdes J. The Ki-67 protein: from the known and the unknown. J Cell Physiol 2000;182:311-322.

10. Spruck III CH, Rideot III WM, Olumi AF, et al. Distinct pattern of p53 mutations in bladder cancer: Relationship to tobacco usage. Cancer Res 1993;53:1162-1166.

11. Kim EJ, Kim YJ, Jeong P, et al. Methylation of the RUNX3 promoter as a potential prognostic marker for bladder tumor. J Urol 2008; 180:1141-1145.

12. Solomides CC, Birbe RC, Nicolaou N, et al. Does mitosis-specific marker phosphohistone $\mathrm{H} 3$ help the grading of upper tract urothelial carcinomas in cell blocks? Acta Cytol 2012;56:285-288.

13. Gunia S, Kakies C, Erbersdobler A, et al. Scoring the percentage of Ki67 positive nuclei is superior to mitotic count and the mitosis marker phosphohistone $\mathrm{H} 3(\mathrm{PHH} 3)$ in terms of differentiating flat lesions of the bladder mucosa. J Clin Pathol 2012;65:715-720.

14. Mangrud OM, Gudlaugsson E, Skaland I, et al. Prognostic comparison of proliferation markers and World Health Organization 1973/2004 grades in urothelial carcinomas of the urinary bladder. Hum Pathol 2014;45:1496-1503.

15. May P, May E. P53 and Cancers. Pathol Biol (Paris) 1995;43:165-173.

16. Malats N, Bustos A, Nascimento CM, et al. P53 as a prognostic marker for bladder cancer: a meta-analysis and review. Lancet Oncol 2005;6:678-686.

17. Abdelzaher E, Kotb AF. High Coexpression of Runt-related Transcription Factor 2 (RUNX2) and p53 Independently Predicts Early Tumor Recurrence in Bladder Urothelial Carcinoma Patients. Appl Immunohistochem Mol Morphol 2016;24:345-354.

18. Scholzen T, Gerdes J. The Ki-67 Protein: From the Known and the Unknown. J Cell Physiol 2000;182:311-322.

19. Santos L, Amaro T, Costa C, et al. Ki-67 Index Enhances the Prognostic Accuracy of the Urothelial Superficial Bladder Carcinoma Risk Group Classification. Int J Cancer 2003; 105:267-272.

20. Kilicli-Camur N, Kilicaslan I, Gulluoglu MG, et al. Impact of p53 and $\mathrm{Ki}-67$ in Predicting Recurrence and Progression of Superficial (pTa and pT1) Urothelial Cell Carcinomas of Urinary Bladder. Pathol Int 2002;52:463-469.

21. Ben Abdelkrim S, Rammeh S, Ziadi S, et al. Expression of topoisomerase II alpha, ki67, and p53 in primary non-muscle-invasive urothelial bladder carcinoma. J Immunoassay Immunochem 2014;35:358-367. 